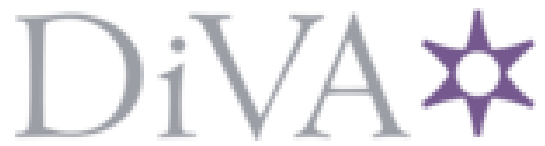

http://www.diva-portal.org

Preprint

This is the submitted version of a paper presented at 25th IEEE International Requirements Engineering Conference Workshops, (REW), Lisbon.

Citation for the original published paper:

Fotrousi, F., Seyff, N., Börstler, J. (2017)

Ethical considerations in research on user feedback

In: Proceedings - 2017 IEEE 25th International Requirements Engineering Conference Workshops, REW 2017 (pp. 194-198). Institute of Electrical and Electronics Engineers Inc.

https://doi.org/10.1109/REW.2017.68

N.B. When citing this work, cite the original published paper.

Permanent link to this version:

http://urn.kb.se/resolve?urn=urn:nbn:se:bth-15609 


\title{
Ethical Considerations in Research on User Feedback
}

\author{
Farnaz Fotrousi ${ }^{1,2}$ \\ farnaz.fotrousi@bth.se
}

\author{
Norbert Seyff ${ }^{2,3}$ \\ norbert.seyff@fhnw.ch
}

\author{
Jürgen Börstler ${ }^{1}$ \\ jurgen.borstler@bth.se
}

\author{
${ }^{1}$ Blekinge Institute of Technology, Karlskrona, Sweden \\ ${ }^{2}$ University of Applied Sciences and Arts Northwestern Switzerland, Windisch, Switzerland \\ ${ }^{3}$ University of Zurich, Zurich, Switzerland
}

\begin{abstract}
Collecting and using user feedback as a method to support requirements engineering, might undermine user rights. This becomes apparent when looking at related areas, e.g., research in user experience, where collecting user feedback also plays an important role. In such settings, researchers need to ensure that the stakeholders' rights and integrity are respected. This paper identifies and discusses some of the ethical challenges and issues a researcher can face, using an example case. Focusing on user feedback, this case can serve as an example for CrowdRE, i.e. several of our findings might apply to CrowdRE in general. However, further research is needed as our work mainly reflects the challenges experienced by the authors of this paper.
\end{abstract}

Index Terms - Ethics, User Feedback, Crowd, Requirement Engineering

\section{I.INTRODUCTION}

User feedback on a software system is one of the available sources for requirements engineering to obtain the overview of stakeholders' ideas and needs [1]. Collecting and utilizing user feedback involve human beings, who often have to interact with technology to accomplish particular tasks and reflect their perceptions or comments. The involvement of human beings implies concerns about user rights.

Research involving humans is typically sensitive regarding ethical aspects and norms [2]. A human being interacting with technology as a part of a research study, implies a set of uncertainties, which makes the researcher taking further ethical considerations [3]. The uncertainties are due to the unpredictable, unforeseen, and unanticipated nature of technology. A new technology, for example using a sensor for data collection, makes the research uncontrollable for human beings and consequently might have abrupt impacts on humans and the environment. Therefore, studies combining humans and technology have particular ethical challenges that a researcher has to deal with.

Researchers collect user feedback mainly in two approaches. In the first approach, the researchers design feedback gathering materials, such as a satisfaction survey form, and select and invite the participants before starting the research. This type of feedback gathering research is not generally dependent on the technology, and the researcher is able to control the feedback gathering focus. In the second approach, user feedback is collected via a feedback tool integrated into a software product or a web page. In the latter approach, users provide their feedback in real-time in the form of complaints, suggestions, new ideas, or praise to reflect on their observations and feelings, while using the product. This approach is dependent on the technology, and the researchers have less control over the feedback gathering process.

A feedback form included in a software product can be triggered by the user by pushing a feedback button to share information/ideas (push feedback mechanism) or can be triggered automatically, while the user is working with the software (pull feedback mechanism). Feedback collected via feedback forms is referred to as explicit feedback [4].

Furthermore, user feedback can be collected indirectly by monitoring user activities that indirectly refer to user preferences. For example, the number of clicks on a specific feature might be an indication of a user's preference or need. The feedback is collected indirectly via monitoring the user activities. Such indirect feedback is referred to as implicit feedback [5]. The activities of users, for example in the software product, or with its connected sensors can be monitored. Analyzing explicit and implicit user feedback together has recently been paid attention to in several studies $[6,7]$. Despite the interest in this research topic, there are few discussions in the literature on challenges and ethical concerns for gathering and using explicit feedback, implicit feedback and the combination of them.

This paper identifies and discusses some of the ethical challenges and issues researchers face, who wish to study implicit and explicit user feedback. We consider the involvement of a crowd to provide feedback as one possible approach to let a vast group of stakeholders communicate their ideas and needs regarding a software system. However, some of the outcomes of our investigation are also relevant for other CrowdRE approaches. In this paper, we discuss the challenges and issues with the help of an example case.

\section{EXAMPLE CASE}

The following example describes a research case that involves gathering user feedback. The example is used to discuss ethical concerns for conducting such research. The example is partially based on a real case, which was modified to cover broader ethical discussions and to maintain the anonymity of the corresponding researchers. Therefore, the case example is presented with fictive names and details.

"Dr. Julia is a researcher working for a software company in Sweden. The software company has developed a software product that has features connected to sensors. The software 
product is called S-Care and is a system which is used by patients to monitor and process health-related data. The company aims to improve S-Care continuously based on the feedback received from its users. Therefore, user feedback will be collected continuously at least for two years. Julia is a requirements engineering researcher and responsible for the company's research in this field. This includes finding a solution for collecting user feedback in a smart way and using the feedback to identify users' needs for continuous improvement of S-Care. For this purpose, she intends to collect and use both implicit and explicit user feedback.

To do so, Julia selects a feedback tool, developed by a third party, which is integrated with S-Care. This feedback solution is able to gather user feedback continuously and monitor activities of users, while the users are using S-Care or the sensors. The collected feedback via the tool can be in the form of texts, ratings, audios, and annotations of screenshots. The feedback tool supports both push and pull feedback mechanisms. A crowd of almost ten-thousand people represents the users of $S$-Care. Employees within the company developing S-Care are a key user group. Recently, the company has joined a European research project, where the company's aim is aligned with the research project's objective that is about increasing the quality and quantity of collected feedback. Company managers hope the international research project can assist the company in achieving its main aim earlier."

\section{COLLECTING USER FEEDBACK IN RESEARCH}

In this section, we discuss the ethical challenges and considerations of the researcher in the above case (i.e., Julia) for collecting implicit and explicit user feedback. We formulate the challenges of the researcher in the form of ethical questions.

\section{A. Informed Consent}

The ethical dilemmas for a human-technology oriented research such as implicit/explicit feedback gathering are to deal with privacy and confidentiality issues. To address these issues, a researcher needs to obtain informed consent from users. Singer and Vinson [8] discussed that consent is needed if the identity of the user or personal information (e.g., age, gender) is identifiable. If so, a researcher should prepare a consent form that can include elements of disclosure, comprehensions, competence, voluntariness, the actual decisions and the right to withdraw [8]. Normally, before starting a research study, the researcher obtains the full agreement of participants on the elements of informed consent. In many cases, such consent is obtained using a paperbased signature of a participant. In most situations, the users are staying with the research for data collection period, although they could opt-out from the research by communicating with the researcher.

How can the researcher invite the participants for signing a consent form? In the example case discussed in section II, Julia intends to use the personal information of users for the research; therefore, she should provide a consent form about using the feedback tool, where a user needs to sign it in the first usage of the tool digitally. Julia does not have controls in choosing and inviting the participants, in contrast to most other research studies. Participants might not also trust to the research, as they did not meet or being contacted by the researcher in advance. The challenge for Julia is whether she should invite the participants to sign the consent form, for example via an email, or no invitation is needed and the participants, as users of the software have to sign the consent form when they use the feedback tool for the first time. In this report, the participants who signed the consent form for using the software product are referred to as users.

Does consent for the feedback tool need to be merged with the consent for the hosting tool? Although the feedback tool is an individual product developed by a third party, the user perceives the feedback tool as a feature of S-Care. This is because the feedback tool has been integrated with S-Care. Therefore, a question raises whether the consent for the feedback tool should be a part of the consent for S-Care, or be a separate consent. The merged consent brings the risk that a user might withdraw using both applications (i.e., S-Care and the integrated feedback tool), although she might be unsatisfied only with the feedback tool.

How to make consent forms visible to users? In the case example, continuous collection of user feedback for a long period of time, might be a disturbing factor for the users. Therefore, the users should have the rights to withdraw the consent, like in any other research study. However, there is an ethical challenge for Julia on how to make the consent form visible to the users so that they can withdraw them at any time they want. A feature might be designed in the feedback tool to allow the users to withdraw consent. The feature can be implemented in the feedback tool, for example using a deactivation button, to allow users to disable triggering the feedback forms and collecting monitoring data. The question is how to make the withdrawing or deactivating feature visible for users so that it on one side allows the users to withdraw when they want, and on the other side does not act as a stimulus to withdraw. For example, a simple deactivating link at the bottom of the feedback form might encourage the users to get rid of the feedback form forever.

\section{B. Anonymity}

Anonymity is a component of confidentiality involving not collecting any data that can be used to identify subjects; not even names [8]. Binik, et al. [9] encourage respects for individual rights and identify several methods for protecting the anonymity and privacy of users of the Internet.

How to keep the users anonymous in this research? In the example case, Julia intends to collect explicit user feedback anonymously. The anonymous user feedback cannot threaten the employability, reputations and financial situation of the users [10]. The risks might be higher when the users, who provide feedback, are also employees of the company.

However, Julia needs to collect not only explicit user feedback but also implicit user feedback. The implicit feedback is collected by monitoring the user activities in SCare or with the sensors. Such monitoring data could also be used to map the actual observation of the users with their feedback, and, to some extent, enhance internal validity of user feedback research.

Therefore, she has the challenge on how to map collected user feedback with the monitoring data, while the users are 
still anonymous. One solution for her could be to introduce an anonymous user_id. For example device-id of a mobile device, from which the user is connected to the application, might work as an anonymous user_id. Although this user id is somehow anonymous, it may raise an ethical challenge for Julia to assure that to what extent the user is still anonymous. If such monitoring data, for example, could show the location of users in real-time via sensors, an ethical issue regarding privacy is raised. Therefore, depends on the type of monitoring data, Julia needs to investigate to what extent the user is traceable, even though the user_id that is collected anonymously.

Does the power of monitoring data threaten the anonymity? Monitoring user behaviour needs to deal with the ethical dilemmas such as when users know that their behaviours are tracked, what kinds of behaviours are tracked, and how the monitored data is used in research. So, Julia should include in consent when, what, and how the user behaviours are monitored. As long as she is monitoring usage data where it is unlikely that it can be linked to the actual user, the anonymity can be maintained. This, for example, includes monitoring of the user interactions with the software (e.g., the user clicks, features usage). However, there is other data which could be more easily linked to the users and reveal who $\mathrm{s} / \mathrm{he}$ is. For example, monitoring the contents of forms, physical location of users, and sensitive biological data from sensors might threaten anonymity, which could mean that Julia should avoid collecting such data. Furthermore, Julia needs to assess the impact of using the monitoring data on users' trust [11].

Does the technology platform maintain anonymity? To assure that anonymity of users is maintained, Julia has to deal with one of the common technological challenges about security of the platform used in the research. She should assure that in the platform for collecting data including S-Care and user feedback applications, databases, and physical servers suitable protection strategies against security vulnerabilities, disasters, and risks have been considered. Hackers would be a threat for most of software products especially for web- applications [12] to access them via the Internet and decrypt collected data.

Furthermore, as the feedback tool is designed and implemented by a third party, Julia needs to evaluate the feedback tool even via code reviewing. She should assure that data are not stored in another database or the feedback tool does not monitor another data other than what has been planned.

\section{Engaging users to provide feedback.}

In the provided case example in section II, the researcher needs to deal with the following challenges:

How to avoid disturbing users while encourage them to give feedback? As explained in the case example, one of the challenges for Julia is to deal with increasing the quality and quantity of the collected feedback. For example, to increase the quantity of feedback, she activates the automatic triggering of feedback forms. However, such automatic triggering might disturb users and discourage them from sending further feedback or even make them deactivating the feedback tool. Therefore, Julia should investigate and figure out what is the right likelihood, time, and event for triggering feedback that on one side increase the quantity of collected feedback on-time and on the other hand, prevent any ethical issue that causes users' disturbances.
Are incentives for giving feedback ethical? A further challenge for Julia is whether she can use incentives to encourage users in providing feedback. Grant and Sugarman [13] discussed that the use of incentives to recruit and retain users are not always harmful. However, there are types of incentives that should be avoided.

For example, financial incentives need more attention due to economically vulnerable users or undue inducements [14] that affect the quality of collected feedback. However, the incentives that motivate continuous uses of the feedback tool, such as providing feedback to the user feedback (i.e. feedbackto-feedback) could be effective types of incentives.

\section{USING AND REPORTING USER FEEDBACK IN RESEARCH}

This section discusses the ethical challenges and considerations of the researcher (Julia) regarding the example case introduced in section II. We address the ethical challenges regarding the use of feedback for requirements identification as well as reporting the feedback to users. We formulate the challenges of the researcher in the form of ethical questions.

\section{A. Using data in research.}

The researcher needs to deal with the following challenges:

Can user feedback data be used in another research? According to the ACM code of ethics [15], the information collected for a specific purpose cannot be used without further informed consent. Julia needs to write the consent form to explain a reasonable additional long-term use of the data. With the user's consent, as long as the research type has not been changed, she can reuse the collected feedback for a new research study, such as the new EU project. However, she should assure that consent is not generic, because the user cannot imagine the future extends of the research.

Do multiple researchers respect ethical aspects of the project over time? Julia has to collaborate with other researchers in the project, who have also access to the project's data andsmay even intend to store data in various devices. She needs to ensure that ethical considerations in the project, such as protecting personal data, is respected by other researchers as well. As a solution, having an ethical protocol, as a part of the study design, can facilitate ethical treatment of data over time.

\section{B. Informing the users about the results.}

Regarding the provided case example, the researcher needs to answer the following question:

What is the degree of details a researcher should inform the users about the results of the conducted research? It is ethical to inform users about the results of the research and the researchers must be honest in their reports. In reporting the results, distortion, falsification, misleading information or plagiarism should be prevented. But sometimes the disclosure of results might have negative impacts on users [16]. For example, the user might take wrong decisions based on the results without considering the degree of uncertainty in the interpretation of that (especially for health-related results captured by bio-sensors). However, such impact should not 
prevent researchers to share the study results with the users who are interested in receiving it. There should be a balance between the negative impacts and benefits.

\section{Storing user data.}

Researchers are responsible for keeping the collected data safe and secure, and for that purpose, they need to assure that adequate organizational and technical securities are applied. If the data are stored in a remote location like a cloud, the researchers should ensure conventional security measures such as firewalls and passwords have been accurately applied. If the user feedback is stored in a cloud, the researcher needs to investigate the trustworthiness of the technologies that the cloud provider is using.

Which storage is ethical to keep the user feedback? Julia has to collect many user feedback for at least two years. Keeping a big data storage for long-time might be a challenge for her. Maintaining data in a third-party location might be another solution. Especially for qualitative user feedback, where a stricter archiving policy, rather than quantitative monitoring data, is needed [17]. However, due to the EU norm [18], Julia should assure that data are kept inside EU, and the storage technology of the third-party is trustable.

What will happen to the user feedback after the study is completed? It is important for Julia to plan for how long the company should retain the user feedback. Julia needs to identify what will happen after the study has been completed. For example, what data will be stored, where they will be stored and who will be responsible for them. She needs to explain the chosen policy of the company, when preparing consent. The retaining period has been regulated in some disciplines. For example, according to US regulation, health data should be kept for at least 25 years. This retaining period might also be applied to user feedback and monitoring data, if the sensors are collecting data about users' health.

\section{CONCERNS REGARDING INTERNATIONALIZATION}

Laws and rules are establishing guidelines for decision-makers about ethical aspects. However, ethical norms in society are sets of moral choices that are also impacted by culture [19]. The ethical norms tend to be broader and informal than rules, although most societies use laws to enforce accepted moral standards and ethical rules [10]. This brings a challenge for a researcher, such as Julia, who works in studies involving human beings in more than one country. Therefore, she needs to respect the moral choices in the corresponding societies and understand the "local" values [20], which might trouble her with taking ethical decisions.

Furthermore, sometimes the researcher needs to resolve the conflicts between rules applicable for a research project. For example, European and Russian laws both force to keep personal data in the same territory that data is collected. This brings the challenge for the researcher how to resolve such conflicts.

Ethical disagreements and conflicts can be consulted with advisory community boards within or across countries. For example, the European Network of Research Ethics CommitteesEUREC (http://www.eurecnet.org/index.html) brings together already existing National Research Ethics Committees (RECs) associations, networks or comparable initiatives on the European level. EUREC creates a good infrastructure for mutual exchanging of RECs in Europe in the field of research involving human participants. The RECs review and consult research projects and guide them in unethical aspects in designing research projects.

\section{CONCLUSION}

Research involving humans is a sensitive type of studies, which is mostly recommended to be reviewed by an ethics committee to ensure that the appropriate ethical standards are met. This paper discussed ethical issues, concerns, and challenges in the research on explicit and implicit user feedback, which involves both human and technology. In this type of study, human interacts with a software product and give feedback. Furthermore, their interaction with the software is monitored. This approach is one instance of a CrowdRE approach and several of the issues and challenges discussed are also true for other approaches where a large (anonymous) crowd is involved in requirements engineering activities.

This paper discussed the important ethical aspects of this type of research including privacy and confidentiality issues. Consent forms and anonymity in collecting feedback as well as using, storing and reporting data in research were some of the topics addressed.

\section{REFERENCES}

[1] E. C. Groen, N. Seyff, R. Ali, F. Dalpiaz, J. Doerr, E. Guzman, et al., "The Crowd in Requirements Engineering: The Landscape and Challenges," IEEE software, vol. 34, pp. 44-52, 2017.

[2] B. D. Sales and S. E. Folkman, Ethics in research with human participants: American Psychological Association, 2000.

[3] P. Sollie, "Ethics, technology development and uncertainty: an outline for any future ethics of technology," Journal of Information, Communication and Ethics in Society, vol. 5, pp. 293-306, 2007.

[4] W. Maalej, H.-J. Happel, and A. Rashid, "When users become collaborators: towards continuous and context-aware user input," in Proceedings of the 24th ACM SIGPLAN conference companion on Object oriented programming systems languages and applications, 2009, pp. 981-990.

[5] D. Kelly and J. Teevan, "Implicit feedback for inferring user preference: a bibliography," ACM SIGIR Forum, vol. 37, pp. 18-28, 2003.

[6] G. Jawaheer, P. Weller, and P. Kostkova, "Modeling user preferences in recommender systems: A classification framework for explicit and implicit user feedback," $A C M$ Transactions on Interactive Intelligent Systems (TiiS), vol. 4, pp. 8:1- 8:26, 2014.

[7] F. J. Martin, J. Shur, D. Brown, A. Ashenfelter, K. Baldwin, and S. Taylor, "Systems and methods for providing personalized recommendations of products and services based on explicit and implicit user data and feedback," ed: Google Patents, 2009.

[8] J. Singer and N. G. Vinson, "Ethical issues in empirical studies of software engineering," IEEE Transactions on Software Engineering, vol. 28, pp. 1171-1180, 2002.

[9] Y. M. Binik, K. Mah, and S. Kiesler, "Ethical issues in conducting sex research on the Internet," Journal of Sex Research, vol. 36, pp. 82-90, 1999. 
[10] D. Gajjar, "Ethical consideration in research," Education, vol. 2, 2013.

[11] P. M. Schwartz, "Privacy, ethics, and analytics," IEEE security \& privacy, vol. 9, pp. 66-69, 2011.

[12] H. E. Keller and S. Lee, "Ethical issues surrounding human participants research using the Internet," Ethics \& behavior, vol. 13, pp. 211-219, 2003.

[13] R. W. Grant and J. Sugarman, "Ethics in human subjects research: do incentives matter?," Journal of Medicine and Philosophy, vol. 29, pp. 717-738, 2004.

[14] C. L. Tishler and S. Bartholomae, "The recruitment of normal healthy volunteers: a review of the literature on the use of financial incentives," The Journal of Clinical Pharmacology, vol. 42, pp. 365-375, 2002.

[15] C. M. ACM, "ACM code of ethics and professional conduct. Adopted by ACM Council (10/16/92)." 1992.
[16] C. V. Fernandez, E. Kodish, and C. Weijer, "Informing study participants of research results: an ethical imperative," IRB: Ethics \& Human Research, vol. 25, pp. 12-19, 2003.

[17] O. Parry and N. S. Mauthner, "Whose data are they anyway? Practical, legal and ethical issues in archiving qualitative research data," sociology, vol. 38, pp. 139-152, 2004.

[18] C. A. C. Oliveira, E. Del Hoyo-Barbolla, A. Demoisy, A. Giovanetti, W. Hannak, J. Houghton, et al., "Data protection and privacy ethical guidelines, Ethical Review in FP7," 2009.

[19] S. J. Vitell, S. L. Nwachukwu, and J. H. Barnes, "The effects of culture on ethical decision-making: an application of Hofstede's typology," in Citation Classics from the Journal of Business Ethics, ed: Springer, 2013, pp. 119-129.

[20] S. R. Benatar, "Towards progress in resolving dilemmas in international research ethics," The Journal of Law, Medicine \& Ethics, vol. 32, pp. 574-582, 2004. 